\title{
Bem-estar subjetivo: um estudo com adolescentes trabalhadores ${ }^{1}$
}

\author{
Adriane Xavier Arteche ${ }^{2}$ \\ Denise Ruschel Bandeira ${ }^{3}$
}

\begin{abstract}
Resumo
A presente pesquisa avaliou o bem-estar subjetivo, comparando adolescentes em dois distintos regimes de trabalho e adolescentes não trabalhadores. A amostra foi composta de 193 jovens (77 não trabalhadores, 58 trabalhadores em regime regular e 58 trabalhadores em regime educativo) entre 14 e 17 anos de idade. Os instrumentos utilizados foram um questionário demográfico, a Escala Multidimensional de Satisfação de Vida, as Escalas de Afeto Positivo e Afeto Negativo e a Escala de Eventos Estressores. Os resultados mostraram que o trabalho na adolescência pode ser positivo, especialmente para adolescentes em regime de trabalho educativo, já que se mostraram mais satisfeitos com suas vidas, principalmente em relação à subescala self comparado. Na comparação entre os dois grupos de jovens trabalhadores, aqueles de regime educativo mostraram-se mais satisfeitos com seu trabalho.

Palavras-Chave: Satisfação de vida; Afeto; Projetos sociais.
\end{abstract}

\section{Subjective well-being: A study with working adolescents}

\begin{abstract}
The present research assessed the subjective well-being, comparing youngsters employed in two different frameworks and non-workers. The participants were 193 adolescents living in poverty, aged from14 to 17 years (77 non-workers, being that 58 were working in the regular system, and 58 employed in an educational system). The instruments used were a demographic questionnaire, the Multidimensional Life Satisfaction Scale, the Positive and Negative Affect Scale and the Stressful Situations Scale. The data indicated that work in adolescence can be positive, especially if in an educational system. The results showed that adolescents working in educational programs were more satisfied with their lives than the others. Also, they showed more satisfaction with their work than the adolescents employed in the regular system.

Keywords: Life satisfaction; Affect; Social projects.
\end{abstract}

\section{Introdução}

Desde os antigos filósofos, a felicidade tem sido considerada a grande meta e um elemento motivacional do ser humano. A partir do final da década de 90, no entanto, estudos clássicos que vinham sendo desenvolvidos desde a década de 70 passaram a ser questionados. Estimulados pelo advento da Psicologia Positiva, que propõe a modificação do foco da psicologia de uma reparação das coisas ruins da vida para a construção de qualidades positivas (Fredrickson, 2001; Sheldon \& King, 2001), estudiosos sugeriram que o bem-estar subjetivo necessariamente inclui elementos positivos (Diener, Suh, Lucas \& Smith, 1999).

Desde esta perspectiva, o bem-estar subjetivo passou a ser compreendido como uma variável multifacetada, composta tanto de elementos positivos quanto negativos (Diener, Suh, Lucas \& Smith, 1999). O modelo explicativo mais aceito atualmente, inclusive para crianças (Huebner \& Dew, 1996) e adolescentes
(Wilkinson \& Walford, 1998), entende esse construto como sendo composto por três fatores: satisfação de vida (componente cognitivo), afeto positivo e afeto negativo (componentes afetivos). Cada um destes pode ainda ser subdividido em subfatores, como os domínios casamento e amizade, na satisfação de vida, e as emoções alegria e afeição, no afeto positivo (Diener, Suh \& Oishi, 1997).

Paralelamente aos estudos sobre fatores que compõem o bem-estar, os pesquisadores estiveram interessados em conhecer a interação desse construto com outras variáveis. Quando pesquisas revelaram que variáveis demográficas, como nível de instrução e classe econômica, respondiam por um pequeno percentual da variância do bem-estar (Diener, 1984; Diener, Suh \& Oishi, 1997), o interesse dos pesquisadores voltou-se então para as outras variáveis que poderiam estar correlacionadas com o bem-estar, como com a auto-estima.

Quando se trata de analisar as relações entre auto-estima e bem-estar, as opiniões são controversas.

${ }^{1}$ Projeto financiado pela CAPES

Endereço para correspondência:

${ }^{2}$ E-mail: darteche@terra.com.br

${ }^{3}$ E-mail: drbandei@terra.com.br 
Enquanto Ryff (1989), por exemplo, indica que a autoestima é um dos componentes do bem-estar, mais especialmente da satisfação de vida, outros autores (Bergman \& Scott, 2001; Lucas, Diener \& Suh, 1996; Yarcheski, Mahon \& Yarcheski, 2001) entendem esta variável como independente, embora correlacionada com bem-estar.

Apesar de tais divergências, nem todos os resultados das pesquisas sobre bem-estar são controversos. Pesquisadores têm sido unânimes ao indicar que adolescentes, em medidas unidimensionais do construto, reportam bons níveis de bem-estar subjetivo (Diener, 1996; Wagner, Ribeiro, Arteche \& Bornholdt, 1999), embora estes bons níveis de bemestar possam não ser tão elevados quando se avalia o bem-estar em relação aos diferentes contextos da vida do adolescente, por meio de medidas multidimensionais que consideram, por exemplo, o nível de bem-estar em relação à família, à amizade e à escola. A maior parte dos estudos sobre jovens, inclusive aqueles que têm como objetivo avaliar o bem-estar, centram-se nestes contextos tradicionais da vida do adolescente, como família, escola e relacionamentos. A realidade de violência, pobreza e limitadas oportunidades de futuro, no entanto, introduz um novo cenário na vida de grande parte dos jovens brasileiros. São inúmeros meninos e meninas cujo maior conflito e foco de preocupação durante a adolescência, segundo Oliveira e Costa (1997), não é o estudo, o lazer, a escolha de festas ou a administração do tempo livre - como para os jovens de classe média - mas, sim, o trabalho.

Como resultado do exercício de tarefas inadequadas às demandas desta etapa da vida, alguns estudos realizados têm encontrado resultados negativos quanto ao impacto do trabalho no desenvolvimento dos adolescentes (Greenberger, Steinberg \& Vaux, 1981; Steinberg \& Dornbusch, 1991), especialmente no bemestar. Greenberger e cols. (1981), em um estudo com 531 adolescentes americanos, encontraram que a sobreposição entre o estresse provocado pelo trabalho e as angústias típicas da adolescência foi considerada a responsável pelo menor nível de bem-estar dos trabalhadores.

Ainda assim, a simples proibição do trabalho para adolescentes não ameniza os problemas. No Brasil, jovens desempregados apresentam menores níveis de saúde e auto-estima que aqueles empregados ou somente estudantes (Sarriera, 1993), sendo o desemprego considerado um importante preditor de baixos níveis de bem-estar (Diener, 1984) também entre adolescentes (Goede, Sprulit \& Maas, 1999; Sarriera, Berlim, Verdin \& Câmara, 2000). Alguns autores indicam que, embora o trabalho juvenil seja, muitas vezes, prejudicial, é ele que está dando aos sujeitos status social (Hansen \& Jarvis, 2000) e o reconhecimento como agentes produtivos (Bonamigo, 1996). Tal dado leva-nos a refletir sobre a impossibilidade de somente impedir que os adolescentes trabalhem. $\mathrm{Na}$ busca por soluções para essa questão, alguns aspectos legais têm sido reexaminados, especialmente na última década. Com base no Estatuto da Criança e do Adolescente (1990), foi introduzida uma modalidade de trabalho chamada trabalho-educativo, compreendida como aquela na qual as exigências pedagógicas prevalecem sobre a questão produtiva.

A maior parte dos estudos no Brasil - e no exterior - com adolescentes trabalhadores desconsidera a existência de diferentes regimes de trabalho e busca comparar apenas os jovens que trabalham com os que não trabalham (Greenberger, Steinberg \& Vaux, 1981; Sarriera, 1993). Um estudo realizado por Hansen e Jarvis (2000), no entanto, salientou que diferentes contextos de emprego promovem diferentes repercussões no desenvolvimento do adolescente. $\mathrm{Na}$ presente pesquisa foram considerados dois grupos distintos de adolescentes trabalhadores: adolescentes trabalhadores em regime educativo, inseridos em projetos sociais; e jovens trabalhadores regulares, considerados, para este estudo, adolescentes que exercem atividade economicamente remunerada na economia regular ou irregular, legais ou não no exercício de suas funções. Foram ainda participantes do estudo, servindo como grupo de comparação, adolescentes não trabalhadores.

Considerando, assim, as diferentes situações ocupacionais dos adolescentes, a importância do contexto trabalho para os mesmos e a variável bemestar subjetivo, o presente estudo teve como objetivo geral avaliar o bem-estar subjetivo em adolescentes trabalhadores em dois distintos regimes laborais e em não trabalhadores. Com base nas possíveis diferenças e similaridades entre os grupos, os objetivos específicos deste estudo foram verificar se existiam diferenças em relação aos níveis de bem-estar (satisfação de vida, afeto positivo e afeto negativo) dos três grupos de adolescentes e verificar qual o efeito das variáveis demográficas, média de eventos estressores e média de afeto (positivo e negativo) sobre a satisfação de vida do adolescente.

\section{Método}

\section{Participantes}

A amostra deste estudo foi constituída de 193 adolescentes, de ambos os sexos, 112 meninos (58\%) e 81 meninas (42\%), com idades entre 14 e 17 anos (média de 15,7 anos, $d p=1,0$ ano), que freqüentavam escolas públicas da cidade de Porto Alegre (escolaridade média 7,7 série, $d p=1,6)$. Os participantes foram divididos em três grupos, conforme a situação laboral, assim denominados: $\mathrm{TE}=$ adolescentes trabalhadores 
em regime de trabalho educativo $(n=58), \mathrm{TR}=$ adolescentes trabalhadores em regime regular $(n=58)$ e NT $=$ adolescentes não-trabalhadores $(n=77)$.

\section{Instrumentos}

Foi aplicado o Questionário de Dados SocioDemográficos, composto de 16 perguntas fechadas de múltipla escolha. Além disso, foi aplicada a Escala Multidimensional de Satisfação de Vida Infantil (Giacomoni, 2002) composta de 57 itens (seis fatores e uma medida de avaliação global), dispostos em uma escala Likert de cinco pontos. Para o presente estudo, os itens inadequados à amostra de adolescentes foram adaptados, bem como foram incluídos itens relativos à dimensão trabalho, para os grupos TE e TR. A versão da escala adaptada para adolescentes - aqui denominada de EMSV-A - após a análise fatorial, contou com 61 itens em seis fatores: família $(\alpha=0,90)$, self comparado $(\alpha=0,85)$, amizade $(\alpha=0,81)$, escola $(\alpha=0,75)$, nãoviolência $(\alpha=0,73)$, self $(\alpha=0,81)$, trabalho $(\alpha=0,78)$ e uma subescala global $(\alpha=0,84)$. Para fins de análise dos dados, foi elaborada uma medida denominada Total $(\alpha=0,90)$, que inclui todas as subescalas da EMSV-A, com exceção da subescala global.

Foram ainda aplicadas as Escalas PANAS de afeto positivo e afeto negativo cujas versões brasileiras foram construídas por Giacomoni e Hutz (1997) baseadas nas escalas PANAS americanas (Watson, Clark \& Tellegen, 1988). No presente estudo, as Escalas PANAS, após a análise fatorial, finalizaram com 46 itens, sendo 24 descritores de Afeto Negativo $(\alpha=0,92)$ e 22 descritores de Afeto Positivo $(\alpha=0,88)$.

Por fim, foi utilizada neste estudo a Escala de Eventos de Vida Estressores em Adolescentes (Ferlin, Lima, Alchieri, Kristensen \& Flores, 2000), que possui 62 itens na forma de eventos de vida estressores, com avaliação de sua ocorrência e intensidade. Nesta pesquisa, foi considerada somente a soma de eventos estressores ocorridos.

\section{Procedimentos}

Para o grupo de jovens de trabalho educativo foi realizado o contato com as instituições de projetos sociais e o posterior contato com os adolescentes, apresentando o estudo e solicitando consentimento informado dos responsáveis. A seguir, foi realizada, pela pesquisadora e um auxiliar de pesquisa, a aplicação coletiva dos instrumentos na seguinte ordem: questionário de dados sociodemográficos, EMSV, Escalas PANAS e Escala de Eventos Estressores. Para obter a amostra de adolescentes que trabalhavam em regime regular e de adolescentes que não trabalhavam foi feito o contato com escolas públicas que ofereciam curso noturno. Foi explicado o trabalho para todos os alunos das turmas noturnas e estes foram convidados a preencher uma ficha de identificação, com base na qual foram selecionados aqueles jovens que preenchiam os critérios da pesquisa referentes à idade e situação ocupacional. No segundo momento, aqueles que cumpriram os requisitos foram então convidados a participar efetivamente do estudo, tendo sido solicitados a fornecer o consentimento informado. Em uma terceira etapa, os adolescentes que se dispuseram a ser participantes foram então reunidos em grupos e, em horário de aula, foram aplicados os instrumentos, na mesma ordem estabelecida para os jovens trabalhadores em regime educativo.

\section{Resultados}

A fim de atingir o primeiro objetivo específico do estudo (verificar se existiam diferenças entre os níveis de bem-estar dos três grupos), inicialmente foram realizadas as análises referentes às propriedades psicométricas das escalas utilizadas, a fim de confirmar a validade dessas medidas para a avaliação do construto na presente amostra. A seguir foram realizadas as médias e as análises de comparação entre os grupos. Os resultados serão apresentados conforme cada um dos instrumentos.

No que diz respeito às Escalas PANAS, utilizadas para avaliar os componentes afetivos do bem-estar - afeto positivo e afeto negativo - após a realização da Análise Fatorial Confirmatória, por meio do método dos componentes principais com rotação Varimax e das análises de consistência interna, foi realizada uma análise de correlação entre as escalas. Conforme esperado, a correlação não foi significativa $(r=0,12)$.

A seguir foram calculadas as médias de cada uma das escalas. O escore médio do fator afeto positivo foi superior ao de afeto negativo, apresentando médias de 3,04 $(d p=0,64)$ e 1,96 $(d p=0,69)$, respectivamente. Tais índices, transpostos para a pontuação da escala Likert, mantém-se entre mais ou menos e bem pouco para afeto positivo e bem pouco e nenhum pouco para afeto negativo. Foram também realizadas as médias e desvios padrão por grupo. Tais dados podem ser observados na Tabela 1. 
Tabela 1 - Médias e Desvios Padrões de Afeto Positivo e Afeto Negativo por Grupo

\begin{tabular}{lccccc}
\hline & \multicolumn{2}{c}{ Afeto Positivo } & \multicolumn{3}{c}{ Afeto Negativo } \\
Variáveis Independentes & $N$ & $M$ & $\mathrm{dp}$ & $M$ & $\mathrm{dp}$ \\
\hline Trabalho Educativo & 58 & 3,11 & 0,68 & 2,03 & 0,65 \\
Trabalho Regular & 58 & 3,06 & 0,66 & 2,00 & 0,73 \\
Não Trabalha & 77 & 2,97 & 0,64 & 1,88 & 0,69 \\
\hline
\end{tabular}

Buscando avaliar possíveis diferenças entre tais medidas nos três grupos - TE, TR e NT - foi realizada uma ANOVA, que apontou que, embora exista uma tendência dos adolescentes de TE de reportarem maiores níveis de afeto, tanto positivo quanto negativo, e dos adolescentes de NT reportarem os menores níveis de afeto, as diferenças entre os grupos não foram significativas [afeto positivo: $\mathrm{F}(2,190)=0,82$; afeto negativo: $\mathrm{F}(2,190)=0,94]$.

No que diz respeito à avaliação da satisfação de vida, a Análise Fatorial Confirmatória, por meio do método dos componentes principais, com rotação Direct Oblimin, revelou a manutenção dos seis fatores que compõem a escala total, quais sejam, família, self comparado, escola, não-violência, amizade e self. $\mathrm{O}$ índice de consistência interna da escala total (49 itens) foi de 0,92 e a variância explicada foi 49,7\%. Outra análise fatorial foi realizada somente para os grupos TE e TR, incluindo os cinco itens referentes à satisfação de vida no trabalho. Estes formaram, conforme esperado, um novo fator, mantendo quase o mesmo índice de consistência interna da escala total $(\alpha=0,90)$, mas aumentando a variância explicada para 50,9\%.

Considerando que todas as subescalas deveriam avaliar dimensões de um mesmo construto - satisfação de vida - foi realizada uma análise de correlação entre as subescalas. Além disso, buscou-se verificar as correlações das subescalas com a escala total (soma dos seis fatores da escala original). A Tabela 2 apresenta tais resultados.

Tabela 2 - Coeficientes de Correlações entre as subescalas Global, Trabalho, Família, SelfComparado, Escola, NãoViolência, Amizade, Selfe Total

\begin{tabular}{lcccccccc}
\hline & Global & Trabalho & Família & $\begin{array}{c}\text { Self } \\
\text { Comparado }\end{array}$ & Escola & $\begin{array}{c}\text { Não- } \\
\text { violência }\end{array}$ & Amizade & Self \\
\hline $\begin{array}{l}\text { Trabalho } \\
\text { Família }\end{array}$ & $0,42^{* *}$ & - & - & & & & & \\
Self & $0,61^{* *}$ & $0,35^{* *}$ & - & & & & & \\
Comparado & 0,008 & $-0,04$ & 0,03 & - & & & & \\
$\begin{array}{l}\text { Escola } \\
\text { Não- }\end{array}$ & $0,36^{* *}$ & $0,27^{* *}$ & $0,32^{* *}$ & 0,04 & - & & & \\
violência & $0,24^{* *}$ & $0,19^{*}$ & $0,28^{* *}$ & 0,02 & $0,21^{* *}$ & - & & \\
Amizade & $0,50^{* *}$ & $0,27^{* *}$ & $0,49^{* *}$ & 0,03 & $0,31^{* *}$ & $0,26^{* *}$ & - & \\
Self & $0,60^{* *}$ & $0,35^{* *}$ & $0,49^{* *}$ & 0,10 & $0,43^{* *}$ & $0,24^{* *}$ & $0,54^{* *}$ & - \\
Total & $0,65^{* *}$ & $0,35^{* *}$ & $0,74^{* *}$ & $0,41^{* *}$ & $0,53^{* *}$ & $0,49^{* *}$ & $0,73^{* *}$ & $0,72^{* *}$ \\
\hline
\end{tabular}

Nota: $* *$ correlações significativas com $\mathrm{p}<0,01,{ }^{*}$ correlação significativa $\operatorname{com} \mathrm{p}<0,05$

Diferentemente do esperado, a subescala self comparado não se correlacionou com nenhuma das demais subescalas, com exceção da total $(\mathrm{r}=0,41$; $\mathrm{p}<0,01)$. Com o intuito de verificar se esta correlação representava efetivamente uma relação entre essa subescala e o construto satisfação de vida avaliado na escala total, seus itens foram retirados da soma total. Refeitas as correlações, os resultados demonstraram que a subescala self comparado não mais se correlacionou de forma significativa com a escala total $(\mathrm{r}=0,06)$.

Ainda no que diz respeito aos resultados descritivos da EMSV-A, a fim de verificar a média de bem-estar na presente amostra, foram realizadas as médias para cada uma das subescalas, bem como para a escala total, considerando-se também os três grupos de adolescentes. A Tabela 3 apresenta tais resultados. 
Tabela 3 - Médias e Desvios Padrões da Escala Multidimensional de Satisfação de Vida

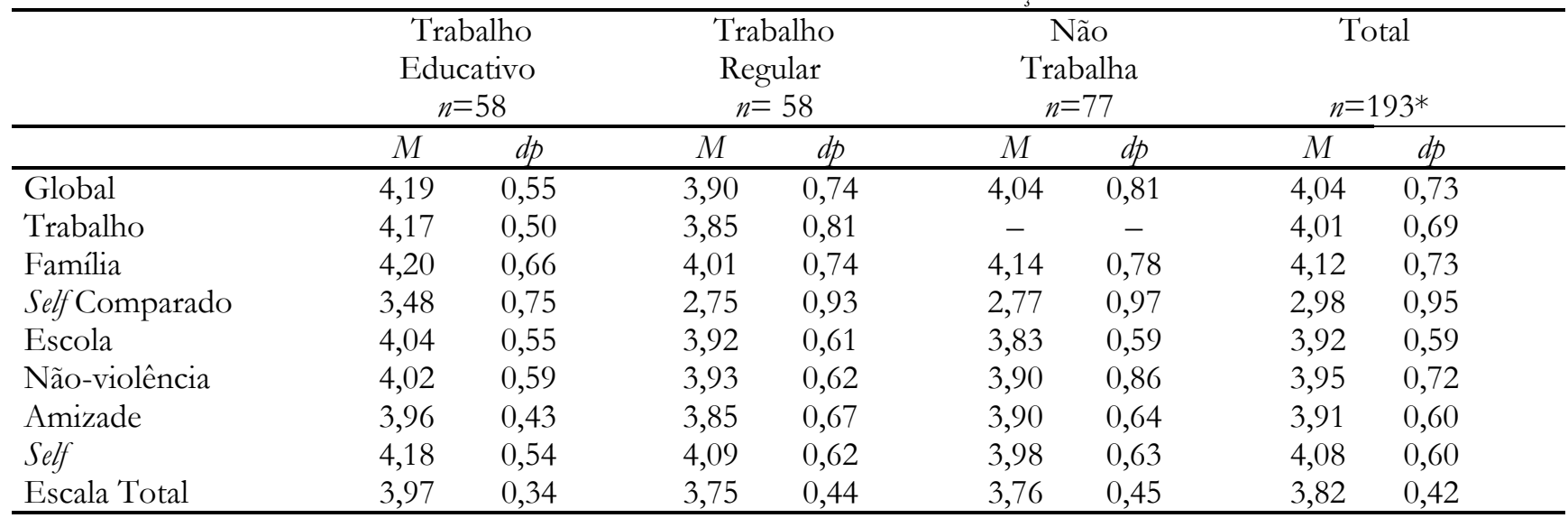

*Nota: Na soma total da subescala Trabalho $n=116$

Pode-se observar que os adolescentes reportaram bom níveis de bem-estar, visto que as médias das subescalas e da escala total ficaram acima de 3,75, com exceção da subescala self comparado. A fim de verificar se as médias dos grupos eram significativamente diferentes, foram realizadas ANOVAS para todas as subescalas, com exceção da subescala trabalho. Os resultados indicaram que apenas a média da subescala self comparado diferencia os grupos, sendo que o TE apresentou uma média significativamente superior aos dois outros grupos $[\mathrm{F}(2,190)=12,67$, $p<0,01]$. Na escala total, o TE também apresentou uma média significativamente superior aos dois outros grupos $[\mathrm{F}(2,190)=5,25, \quad p<0,01]$, entretanto, tal diferença deve-se à subescala de self comparado, uma vez que, quando se retiram os itens dessa subescala da escala total, desaparece essa diferença.
Em relação à subescala trabalho, a fim de verificar possíveis diferenças entre TE e TR, foi realizado o teste $t$. $\mathrm{O}$ resultado indicou que os adolescentes do TE apresentaram uma média significativamente superior aos adolescentes do TR nesta subescala $(\mathrm{t}=2,48 ; g l=114 ; p<0,05)$.

Buscando esclarecer o resultado não esperado da não-correlação entre self comparado e as demais dimensões, foi realizada uma análise de correlação entre a EMSV-A e as Escalas PANAS. Caso a subescala self comparado realmente representasse uma das dimensões da satisfação de vida, esperava-se uma correlação com, pelo menos, uma das dimensões afetivas. Entretanto, esta subescala foi, novamente, a única que não se correlacionou nem com afeto positivo nem com afeto negativo. Os resultados estão na Tabela 4.

Tabela 4 - Coeficientes de Correlações entre a Escala Multidimensional de Satisfação de Vida e as Escalas PANAS de Afeto Positivo e Afeto Negativo

\begin{tabular}{lcc}
\hline & Afeto Positivo & Afeto Negativo \\
\hline Self Comparado & 0,11 & 0,02 \\
Global & $0,32^{*}$ & $-0,51^{*}$ \\
Trabalho & $0,30^{*}$ & $-0,12$ \\
Família & $0,32^{*}$ & $-0,44^{*}$ \\
Escola & $0,36^{*}$ & $-0,06$ \\
Não-violência & 0,11 & $-0,39 *$ \\
Amizade & $0,32^{*}$ & $-0,32^{*}$ \\
Self & $0,37^{*}$ & $-0,29^{*}$ \\
Total & $0,42^{*}$ & $-0,40^{*}$ \\
\hline
\end{tabular}

Nota: ${ }^{*}$ correlações significativas com $\mathrm{p}<0,01$

Por fim, para verificar o efeito conjunto de outras variáveis avaliadas nesta pesquisa sobre a satisfação de vida (segundo objetivo específico) foi realizada uma análise de regressão linear múltipla. A soma total da EMSV-A foi inserida como variável dependente e, como variáveis independentes, grupo, eventos estressores, afeto positivo, afeto negativo e as demográficas morar com a mãe, morar com o pai, escolaridade, sexo, idade. Visto que as variáveis afetivas possuem correlação significativa com a escala total 
(afeto positivo: $\mathrm{r}=0,42 ; \mathrm{p}<0,01$ e afeto negativo: $\mathrm{r}=0,40$; $\mathrm{p}<0,01)$, optou-se pelo método Stepwise, com afeto positivo e afeto negativo inseridos em um segundo momento. O resultado demonstrou que as variáveis demográficas não mostram associação com o escore de satisfação de vida. A inter-relação dos demais fatores explica 47,4\% da variação do nível de satisfação de vida dos participantes. Os resultados podem ser observados na Tabela 5.

Tabela 5 - Resultados da Análise de Regressão da Satisfação de Vida

\begin{tabular}{lccc}
\hline & \multicolumn{3}{c}{ Satisfação de Vida } \\
\cline { 2 - 4 } Variáveis Independentes & $\beta$ & $\mathrm{R}$ & $\mathrm{R}^{2}$ \\
\hline Grupo Trabalho Educativo & 0,26 & 0,24 & 0,05 \\
Eventos Estressores & $-0,08$ & 0,36 & 0,13 \\
Afeto Negativo & $-0,47$ & 0,54 & 0,29 \\
Afeto Positivo & 0,42 & 0,68 & 0,47 \\
\hline
\end{tabular}

\section{Discussão}

A situação laboral é considerada um importante marcador na determinação do curso da adolescência. $\mathrm{Na}$ busca de um olhar mais aprofundado para esta variável, a presente pesquisa buscou verificar como estão se sentindo os adolescentes estudantes em situação de risco social, tanto aqueles que não trabalham, quanto aqueles que trabalham em empregos regulares e em projetos sociais, no regime de trabalho educativo. $\mathrm{O}$ perfil de cada um destes três grupos será discutido a seguir, destacando-se os aspectos que se revelaram comuns e aqueles que diferenciaram os jovens participantes deste estudo em relação ao bem-estar subjetivo.

A condição de não trabalhar não fez com que os jovens não trabalhadores (NT) participantes deste estudo obtivessem menores níveis de satisfação de vida do que aqueles trabalhadores (TE e TR). Os resultados, que apontaram para bons níveis de bem-estar nos três grupos, corroboram os achados da literatura, que indica que adolescentes reportam, em geral, bons níveis de bem-estar subjetivo (Diener, 1996; Wagner, Ribeiro, Arteche \& Bornholdt, 1999) e contraria os resultados obtidos por Sarriera (1993), em cujo estudo os jovens não trabalhadores apresentaram menores níveis de bem-estar. $\mathrm{Na}$ presente pesquisa os adolescentes não trabalhadores, assim como os jovens do TR, apresentaram média mais baixa apenas na subescala self comparado. Uma possível explicação para isso diz respeito às características dos adolescentes em situação de risco. Pode-se pensar que, ao se compararem com outros adolescentes, sentem-se menos satisfeitos. Nesta medida os jovens de TE é que estão conseguindo diferenciar-se dessa realidade.

Os altos escores de satisfação de vida podem ser entendidos com base em três correntes. Uma delas seria o modelo de equilíbrio dinâmico, no qual fatores de personalidade mediariam a linha base de satisfação e, no caso, os eventos vitais e variáveis demográficas moveriam os sujeitos apenas temporariamente acima ou abaixo dessa linha (Diener, 1996; Kahneman, Diener \& Schwarz, 1999). Neste caso, é possível que diferenças nos níveis de satisfação não se devam à situação laboral, mas a fatores como neuroticismo e extroversão, não examinados neste estudo.

Uma segunda explicação seria baseada nos teóricos do desenvolvimento, segundo os quais os adolescentes reportam bons níveis de bem-estar subjetivo (Huebner, Drane \& Valois, 2000) independentemente de configuração familiar, classe econômica, gênero e escolaridade (Diener, 1996; Huebner, Drane \& Valois, 2000). Pode-se pensar que nessa etapa de vida a necessidade de idealização, o elevado nível de fantasia e a urgência em vivenciar novas experiências fazem com que os adolescentes minimizem aspectos negativos de suas vidas. No entanto, cabe ressaltar que as descrições sobre os bons níveis de satisfação dos adolescentes em geral são realizadas não a partir da comparação com amostras de adultos ou de crianças, mas com base no denominado ponto neutro das escalas (respostas "mais ou menos"), acima do qual os adolescentes são referidos como tendo bons níveis de satisfação. Os bons níveis de satisfação podem, assim, não ser uma exclusividade de jovens.

A terceira explicação para os bons níveis de satisfação dos adolescentes do presente estudo advém dos estudos sobre satisfação de vida, auto-estima e comparação social, segundo os quais adolescentes em situação de risco tendem a utilizar-se de um mecanismo de comparação denominado "downward", com cognições do tipo "pelo menos eu tenho uma casa" para se manterem felizes, a despeito da situação social que vivenciam (Wadsworth \& Compas, 2002). Tal teoria poderia explicar também a menor média na subescala self comparado, uma vez que as cognições do tipo downward são hipotéticas, enquanto a subescala self comparado apresenta sentenças específicas que forçam o adolescente a confrontar sua situação com a do outro, inserindo-se na realidade. 
Quaisquer destas explicações, no entanto, não dão conta da influência dos instrumentos utilizados para acessar o bem-estar ou a satisfação de vida nos resultados das pesquisas. É possível também que, pelo fato da satisfação de vida ser uma medida cognitiva, os adolescentes tenham respondido muitas das questões de forma intelectualizada ou socialmente desejada. As respostas intelectualizadas justificariam as diferenças entre os resultados da medida cognitiva (satisfação de vida) e das medidas afetivas, observadas no grupo NT. As médias das escalas PANAS nesse grupo deixam margem para uma reflexão acerca dos resultados referentes ao bem-estar. Os dados revelaram que, conforme o esperado, a média de afeto positivo foi superior à de afeto negativo, corroborando os achados de Huebner e Dew (1996) em um estudo com uma amostra de adolescentes.

No entanto, ainda que a diferença não tenha sido estatisticamente significativa, a média dos jovens do NT foi inferior a dos dois outros grupos, reportando sentirem bem pouco sentimentos como entusiasmo e alegria (afeto positivo) e nem um pouco sentimentos como angústia, raiva e ansiedade (afeto negativo). A baixa média de afeto, especialmente dos nãotrabalhadores, pode significar que os adolescentes não conseguem explicitar ou avaliar medidas afetivas. Talvez, por não trabalhar, sejam emocionalmente mais imaturos. A maior parte dos jovens não trabalhadores freqüentava a escola à noite, assim como os jovens trabalhadores. No entanto, como os primeiros não exercem atividade profissional, é possível que a ausência de atividades extra-escolares propicie uma menor intensidade de vivências afetivas.

É possível, ainda, que este resultado tenha sido observado porque os jovens não trabalhadores convivam apenas em um ambiente (escola) e não sejam estimulados a refletir sobre seus sentimentos como os colegas que trabalham - que são naturalmente mais exigidos quanto a postura profissional e relacionamento interpessoal quando surge algum problema no ambiente de trabalho. Ou ainda, tal dado pode estar indicando uma certa apatia diante das coisas, refletindo uma realidade bastante descrita pelas diretoras, professoras e orientadoras educacionais quando o contato era feito para a realização desta pesquisa. Segundo essas, os jovens do turno da noite são "tanto faz"(sic), ou seja, não se interessam por muita coisa.

Já os adolescentes do TR reportaram escores intermediários entre os grupos TE e NT nas escalas de afeto. Referiram sentir freqüentemente emoções características de afeto positivo e bem pouco emoções típicas de afeto negativo, assim como reportaram que se sentem mais ou menos ou bastante satisfeitos em todas as áreas de suas vidas, com exceção de quando se comparam com outros adolescentes. Tais resultados contrariam premissas da literatura que indicam que a entrada do adolescente no mundo do trabalho traz conseqüências negativas (Greenberger, Steinberg \& Vaux, 1981; Steinberg \& Dornbusch, 1991) e sugerem que, para a amostra estudada, o trabalho não tem sido fator de menor satisfação de vida.

Ao lado disso, os resultados indicam que o trabalho regular pode não ser a melhor alternativa para os jovens em situação de risco. Quando são comparados aos adolescentes de TE, os jovens de TR mostraram-se significativamente menos satisfeitos com o seu trabalho. Pode-se pensar que, ainda que o trabalho regular não tenha feito com que os jovens se sintam menos satisfeitos com suas vidas, mantendo escores altos de satisfação de vida, a realidade do trabalho educativo faz com que os adolescentes sintamse ainda melhor.

O reflexo positivo do trabalho educativo pode ser também observado nos resultados da análise de regressão. Os resultados na análise de regressão linear múltipla, referente ao segundo objetivo específico desta pesquisa, corroboraram os achados da literatura ao explicitar que, na presente amostra, as variáveis demográficas não apresentam associação com o escore de satisfação de vida (Diener, 1984; Diener, Suh \& Oishi, 1997, Huebner, 1991) e, além disso, indicaram que, após as medidas afetivas, afeto positivo e negativo, a variável que mais explicou satisfação de vida foi o grupo de trabalho educativo. Os jovens de TE referiram também sentir mais emoções tanto de afeto positivo quanto de afeto negativo, demonstrando, talvez, uma maior facilidade para avaliarem e expressarem estados afetivos, além de se sentirem bastante satisfeitos com suas vidas. Comparando com os dois outros grupos e respondendo ao objetivo geral deste estudo, os adolescentes de TE mostraram-se mais satisfeitos em todos os aspectos de suas vidas, embora a diferença estatística só tenha se dado nas subescalas trabalho e self comparado. $\mathrm{O}$ fato desses jovens referirem maior satisfação nos seus trabalhos parece ser mais reflexo do próprio ambiente de trabalho do que de ganhos financeiros. Sua remuneração é menor, mas nos projetos, o espaço para a vivência da adolescência é mais respeitado, com momentos de descontração, passeios e brincadeiras.

No que diz respeito à maior média na subescala self comparado, pode-se pensar que os adolescentes de TE são mais estimulados a valorizarem a oportunidade de estar trabalhando e, principalmente, de fazer parte do projeto. Além disso, a avaliação do aproveitamento do jovem não é realizada por notas e provas, mas sim pelo processo pessoal, no qual as conquistas pessoais são extremamente valorizadas. Diante disso, é possível que 
os adolescentes de TE sintam-se melhores quando se comparam aos demais, sendo essa diferença tão importante a ponto da subescala self comparado fazer com que o grupo TE apresentasse média estatisticamente superior aos demais grupos na soma total da escala.

Entretanto, ainda que extrapole os objetivos inicialmente propostos nesta pesquisa, não se pode deixar de considerar um resultado inesperado acerca, especificamente, desta subescala. $\mathrm{O}$ fato da mesma não se correlacionar com nenhuma das demais subescalas, nem com as escalas de afeto, nos leva a pensar que, em adolescentes em situação de risco, o self comparado representa um outro construto, que não satisfação de vida. Mas qual seria esse construto que sofre tal influência do trabalho educativo? Segundo Feinberg, Neiderhiser, Simmens, Reiss e Hetherington (2000), as comparações sociais entre o próprio self e os outros, especialmente familiares e pares da mesma idade, seriam parte do construto auto-estima, variável esta que, de acordo com Lucas, Diener e Suh (1996), forma uma medida independente do bem-estar. Assim, hipotetizase que, na amostra estudada, a subescala self comparado tenha funcionado como uma subescala de auto-estima.

É possível que, em virtude da própria realidade social e familiar em que o jovem está inserido - ainda que trabalhando em um projeto social -, a proposta dos projetos de trabalho educativo não seja suficiente para promover uma mudança na satisfação de vida como um todo, no entanto, tem sido bastante eficaz na satisfação em relação ao trabalho e no incremento da auto-estima. Estudos de validade concorrente entre a subescala self comparado e instrumentos de avaliação de auto-estima, com amostras de adolescentes em situação de risco, são, assim, necessários para a confirmação de tal hipótese.

A possibilidade de comparar adolescentes em diferentes situações ocupacionais permitiu o avanço em relação à maior parte dos estudos que apenas descrevem um grupo, no entanto, visto que o delineamento desta pesquisa utilizou um corte trasnversal, algumas questões ainda permaneceram pendentes, por exemplo: as diferenças apresentadas nesta pesquisa, principalmente entre o grupo de jovens de trabalho educativo e os demais se manteriam após a saída dos mesmos do projeto? Esses jovens já apresentavam, antes de ingressar no projeto, potencialidades ou características que os fizeram apresentar tais resultados?

Acredita-se que a realização de estudos longitudinais, que acompanhem os adolescentes antes mesmo deles ingressarem no mercado de trabalho regular ou educativo, assim como após eles terem saído deste, contribuiriam para uma melhor compreensão das relações entre trabalho e os níveis de bem-estar desses jovens.

\section{Referências}

Bergman, M. \& Scott, J. (2001). Young adolescents' well-being and health-risk behaviours: Gender and socio-economic differences. Journal of Adolescence, 24, 183-197.

Bonamigo, L. (1996). O trabalho e a construção da identidade: um estudo sobre meninos trabalhadores na rua. Psicologia: Reflexão e Crítica, 9(1), 129-152.

Diener, E. (1984). Subjective well-being. Psychological Bulletin, 95(3), 542-575.

Diener, E. (1996). Subjective well-being in crosscultural perspective. Em: H. Grad (Org.). Key issues in cross-cultural psychology: Selected papers from the Twelfh International Congress of the International Association for Cross-Cultural Psychology (p. 319-331). ChampaignUrbana, IL.

Diener, E., Suh, E. \& Oishi, S. (1997). Recent findings on subjective well-being. Indian Journal of Clinical Psychology, 24(1), 25-41.

Diener, E., Suh, E., Lucas, R. \& Smith, H. (1999). Subjective well-being: Three decades of progress. Psychological Bulletin, 125(2), 276-302.

Estatuto da Criança e do Adolescente. (1990). Lei № 8.069, de 13/7/1990. Porto Alegre: CORAG.

Feinberg, M., Neiderhiser, J., Simmens, S., Reiss, D. \& Hetherington, M. (2000). Sibling comparison of differential parental treatment in adolescence: Gender, self-esteem, and emotionality as mediators of the parenting-adjustment association. Child Development, 71(6), 1611-1628.

Ferlin, M., Lima, J., Alchieri, J., Kristensen, C. \& Flores, R. (2000). Desenvolvimento do Inventário de Eventos Estressores na Adolescência (IEEA). Resumos de Comunicações Cientificas, Exponba-se (p. 204). São Leopoldo: Unisinos.

Fredrickson, B. (2001). The role of positive emotions in positive psychology. American Psychologist, 56(3), 218226.

Giacomoni, C. (2002). Bem-estar subjetivo infantil: conceito de felicidade e construção de instrumentos para avaliação. (Tese de Doutorado Não-Publicada). Porto Alegre, RS: Universidade Federal do Rio Grande do Sul - Curso de Pós-Graduação em Psicologia do Desenvolvimento.

Giacomoni, C. \& Hutz, C. (1997). A mensuração do bem-estar subjetivo: escala de afeto positivo e negativo e escala de satisfação de vida [Resumos]. Em Sociedade Interamericana de Psicologia (Org.), 
Anais do XXVI Congresso Interamericano de Psicologia (p. 313). São Paulo, SP: SIP.

Goede, M., Sprulit, E. \& Maas, C. (1999). Individual and family factors and adolescent well-being: A multi-level analysis. Social Behavior and Personality, 27(3), 263-280.

Greenberger, E., Steinberg, L. \& Vaux, A. (1981). Adolescents who work: Health and behavioral consequences of job stress. Developmental Psychology, 17(6), 691-703.

Hansen, D. \& Jarvis, P. (2000). Adolescent employment and psychossocial outcomes. Youth and Society, 31(4), 417-436.

Huebner, E. (1991). Correlates of life satisfaction in children. School Psychology Quaterly, 6, 103-111.

Huebner, E. \& Dew, T. (1996). The interrelationships of positive affect, negative affect and life satisfaction in an adolescent sample. Social Indicators Research, 38, 129-137.

Huebner, E., Drane, W. \& Valois, R. (2000). Levels and demographic correlates of adolescent life satisfaction reports. School Psychology International, 21(3), 281-292.

Kahneman, D., Diener, E. \& Schwarz, N. (1999). Wellbeing: The foundations of hedonic psychology. New York, NY: Russell Sage Foundation.

Lucas, R., Diener, E. \& Suh, E. (1996). Discriminant validity of well-being measures. Journal of Personality and Social Psychology, 71(3), 616-628.

Oliveira, C. \& Costa, A. (1997). Categorias de conflitos no cotidiano de adolescentes mineiros. Psicologia: Reflexão e Crítica, 10(1), 87-104.

Ryff, C. (1989). Happiness is everything, or is it? Explorations on the meaning of psychological wellbeing. Journal of Personality and Social Psychology, 57(6), 1069-1081.
Sarriera, J. (1993). Aspectos psicossociais do desemprego juvenil: uma análise a partir do fracasso escolar para intervenção preventiva. Psico, 24(2), 23-39.

Sarriera, J., Berlim, C., Verdin, R. \& Câmara, S. (2000). Os (des)caminhos dos jovens na sua passagem da escola ao trabalho. Em J. C. Sarriera (Org.). Psicologia Comunitária - Estudos Atuais (p. 45-63). Porto Alegre, RS: Sulina.

Sheldon, K. \& King, L. (2001). Why positive psychology is necessary. American Psychologist, 56(3), 216-217.

Steinberg, L. \& Dornbusch, S. (1991). Negative correlates of part-time employment during adolescence: Replication and elaboration. Developmental Psychology, 27(2), 304-313.

Wadsworth, M. \& Compas, B. (2002). Coping with family conflict and economic strain. Journal of Research on Adolescence, 12(2), 243-274.

Wagner, A., Ribeiro, L., Arteche, A. \& Bornholdt, E. (1999). Configuração familiar e o bem-estar psicológico dos adolescentes. Psicologia: Reflexão e Critica, 12(1), 147-156.

Watson, D., Clark, L., \& Tellegen, A. (1988). Development and validation of brief measures of positive and negative affect: The PANAS scales. Journal of Personality and Social Psychology, 54(6), 1063-1070.

Wilkinson, R. \& Walford, W. (1998). The measurement of adolescent psychological health: One or two dimensions? Journal of Youth and Adolescence, 27(4), 443-453.

Yarcheski, A., Mahon, N. \& Yarcheski, T. (2001). Social support and well-being in early adolescents. Clinical Nursing Research, 10(2), 163-181.

Recebido em setembro de 2003

Reformulado em novembro de 2003 Aprovado em dezembro de 2003

Sobre as autoras:

Adriane Xavier Arteche é psicóloga, mestre em Psicologia do Desenvolvimento, doutoranda em Psicologia do Desenvolvimento pela UFRGS e professora da Universidade Regional Integrada do Alto Uruguai e da Região das Missões - Câmpus Frederico Westphalen.

Denise Ruschel Bandeira é doutora em Psicologia do Desenvolvimento pela UFRGS, professora do Programa de Pós-Graduação em Psicologia da UFRGS e secretária do Instituto Brasileiro de Avaliação Psicológica 
